\section{Distributed Cooperative Control of Hybrid AC/DC Microgrid}

\section{INTRODUCTION}

This paper presents a distributed cooperative control-based (DCC) power management algorithm for a hybrid AC/DC microgrid. The proposed algorithm for a hybrid microgrid system controls the power flow through the interface converter between the AC and DC microgrids. This algorithm allows power sharing between the distributed generators in the microgrid according to their power ratings. Moreover, it enables the fixed scheduled power delivery through the interface converters in both directions at different operating conditions while maintaining voltage regulation and

HYBRID MICROGRID ARCHITECTURE

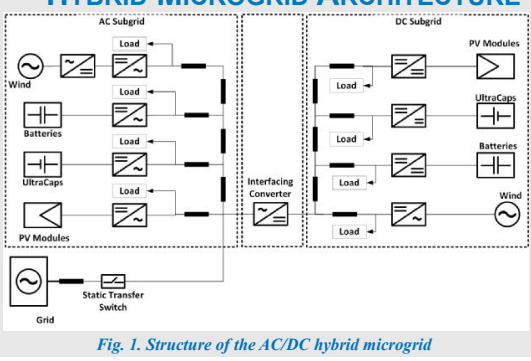

\section{MODES OF OPERATION}

A. Grid-Connected Mode of Operation for Hybrid Microgrid B. Islanded Mode of Operation for Hybrid Microgrid

1. Fixed Scheduled Power through Interface Converter

2. Power Balance in the Subgrids through the Interface Converter

3. Load Shedding

\section{PROPOSED DCC FOR HYBRID AC/DC} MICROGRID

The communications graph developed for the DCC of a hybrid $\mathrm{AC} / \mathrm{DC}$ microgrid is presented in Fig 2

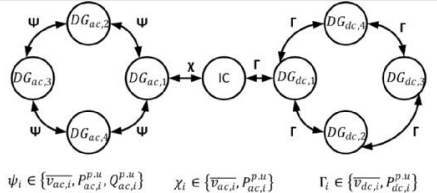

Fig. 2. Communication graph for DCC of hybrid $A C / D C$ microgrid
When power is flowing to the DC subgrid, the reference output voltage of the interface converter is controlled by following equation:

$$
v_{D C}^{I C *}=V_{\text {rated }}+\Delta v_{1}^{I C}+\Delta v_{2}^{I C}
$$

$\Delta v_{1}^{I C} \rightarrow$ regulates the $D C$-side voltage of the interface converter by estimating the average voltage of all nodes of the $D C$ subgrid. The average voltage of the $D C$ subgrid is estimated by the following equation:

$$
\overline{V_{I C}}=V_{I C}+\sum_{j \in N_{D C}} a_{j}\left(\bar{V}_{j}-\overline{V_{I C}}\right)
$$
improving the frequency profile.

\section{PROPOSED DCC FOR HYBRID AC/DC} MICROGRID (CONT'D)

$\Delta v_{2}^{I C} \rightarrow$ is responsible for maintaining the scheduled power through the interface converter or maintaining the same perunit power sharing by all distributed generation in the hybrid microgrid. The update protocol for the second correction factor is illustrated in the following figure:

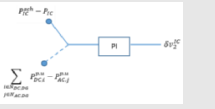

Fig. 3. Calculation of second correction factor

When the interfacing converter is injecting power from the $D C$ subgrid to the AC subgrid, the reference frequency, $\omega^{*}$, is updated according to the flowing equation:

\section{$\omega^{*}=\omega_{\text {rated }}+\delta \omega_{1}^{I C}+\delta \omega_{2}^{I C}$}

\section{SIMULATION RESULTS}

Two case studies were considered to validate the proposed method:

Case 1: Fixed scheduled power flow through the interfacing converter
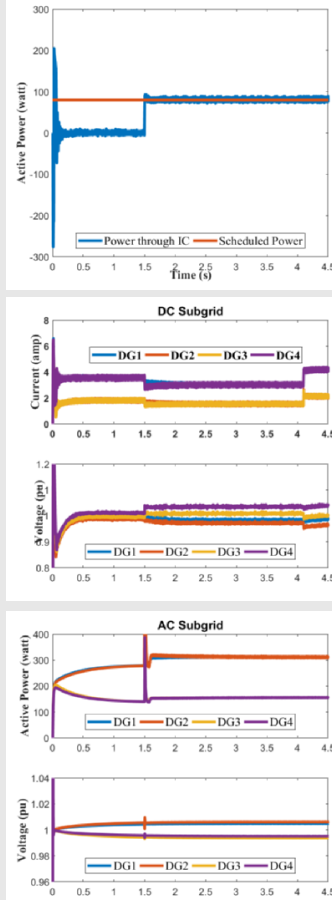

Rasel Mahmud ${ }^{1}$, Arash Nejadpak ${ }^{2}$ ${ }^{1}$ National Renewable Energy Laboratory ${ }^{2}$ University of North Dakota
Case 2: All the distributed generation units in the hybrid $\mathrm{AC} / \mathrm{DC}$ microgrid sharing power according to their ratings
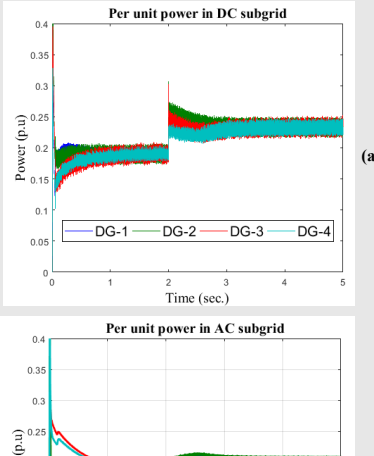

\section{- $\mathrm{DG} 1-\mathrm{DG} 2-\mathrm{DG} 3-\mathrm{DG}$} time (s) Power flow throgh IC

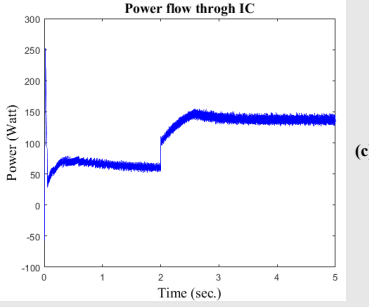

Fig. 5. Performance of the controller to share the load among the distributed generation units in a hybrid microgrid according to the power rating: a) per-unit power of the

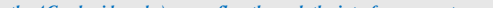

\section{CONCLUSION}

A distributed cooperative control scheme for an AC/DC hybrid microgrid has been proposed. Key features of the proposed algorithm:

$>$ Controls the power flow through the interfacing converter between the $A C$ and $D C$ subgrids.

$>$ The control algorithm uses limited information exchanged with some of the distributed generation in both the $A C$ and $D C$ subgrids to achieve the control objectives, i.e.,

$\square$ Scheduled power flow through the interface converter or

$\square$ Load sharing by the distributed generation units accruing to their ratings.

The simulation results show that the proposed DCC enables control of the power flow through the interface converter in different modes of operation at different operating conditions while maintaining good voltage regulation in both subgrids. 